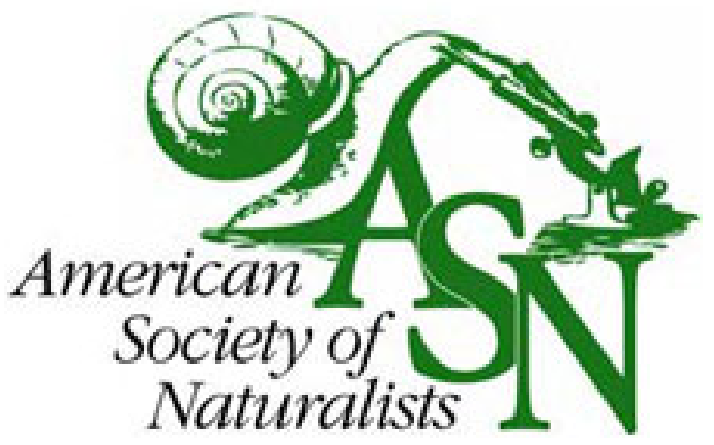

Testing Mechanisms of Bergmann's Rule: Phenotypic Decline but No Genetic Change in Body Size in Three Passerine Bird Populations.

Author(s): Arild Husby, Sabine M. Hille, Marcel E. Visser

Source: The American Naturalist, Vol. 178, No. 2 (August 2011), pp. 202-213

Published by: The University of Chicago Press for The American Society of Naturalists

Stable URL: http://www.jstor.org/stable/10.1086/660834

Accessed: 09/08/2011 03:12

Your use of the JSTOR archive indicates your acceptance of the Terms \& Conditions of Use, available at http://www.jstor.org/page/info/about/policies/terms.jsp

JSTOR is a not-for-profit service that helps scholars, researchers, and students discover, use, and build upon a wide range of content in a trusted digital archive. We use information technology and tools to increase productivity and facilitate new forms of scholarship. For more information about JSTOR, please contact support@jstor.org. 


\title{
Testing Mechanisms of Bergmann's Rule: Phenotypic Decline but No Genetic Change in Body Size in Three Passerine Bird Populations
}

\author{
Arild Husby, ${ }^{1,2, \star}$ Sabine M. Hille, ${ }^{3}$ and Marcel E. Visser ${ }^{4}$ \\ 1. Institute of Evolutionary Biology, School of Biological Sciences, University of Edinburgh, Edinburgh, United Kingdom; \\ 2. Department of Animal Ecology, Evolutionary Biology Centre (EBC), Uppsala University, Nordbyvägen 18D, SE-75236 Uppsala, \\ Sweden; 3. Institute of Wildlife Biology and Game Management, University of Natural Resources and Applied Life Sciences, Gregor \\ Mendel Strasse 33, 1180 Vienna, Austria; 4. Department of Animal Ecology, Netherlands Institute of Ecology (NIOO-KNAW), P.O. Box \\ 50, $6700 \mathrm{AB}$ Wageningen, The Netherlands
}

Submitted November 22, 2010; Accepted March 29, 2011; Electronically published June 24, 2011

Dryad data: http://dx.doi.org/10.5061/dryad.8989.

ABSTRACT: Bergmann's rule predicts a decrease in body size with increasing temperature and has much empirical support. Surprisingly, we know very little about whether "Bergmann size clines" are due to a genetic response or are a consequence of phenotypic plasticity. Here, we use data on body size (mass and tarsus length) from three long-term (1979-2008) study populations of great tits (Parus major) that experienced a temperature increase to examine mechanisms behind Bergmann's rule. We show that adult body mass decreased over the study period in all populations and that tarsus length increased in one population. Both body mass and tarsus length were heritable and under weak positive directional selection, predicting an increase, rather than a decrease, in body mass. There was no support for microevolutionary change, and thus the observed declines in body mass were likely a result of phenotypic plasticity. Interestingly, this plasticity was not in direct response to temperature changes but seemed to be due to changes in prey dynamics. Our results caution against interpreting recent phenotypic body size declines as adaptive evolutionary responses to temperature changes and highlight the importance of considering alternative environmental factors when testing size clines.

Keywords: animal model, climate change, heritability, Parus major, microevolution, selection.

\section{Introduction}

The effects of increase in the global temperature are widespread and diverse, influencing numerous traits in many different taxa (reviewed in Parmesan 2006). One such trait is morphology, and many studies have found that as global temperatures have increased, body size in many bird (e.g., Johnston and Selander 1964; Yom-Tov et al. 2006; Teplitsky

\footnotetext{
* Corresponding author; e-mail: arild.husby@ebc.uu.se.
}

Am. Nat. 2011. Vol. 178, pp. 202-213. (c) 2011 by The University of Chicago. 0003-0147/2011/17802-52631\$15.00. All rights reserved. DOI: $10.1086 / 660834$ et al. 2008) and mammal (e.g., Millien 2004) species has declined. These declines have been suggested to be an adaptation to increasing temperatures and have caused a renewed interest in Bergmann's rule, which states that within a genus of endothermic vertebrates, species occupying warmer geographic regions are smaller than species occupying colder regions (Bergmann 1847; Mayr 1956). Although originally formulated to explain large-scale patterns, Bergmann's rule has since been modified to also concern changes within populations (Mayr 1956). The rule has received much empirical support (Johnston and Selander 1964; Millien and Damuth 2004; Yom-Tov and Yom-Tov 2006; Teplitsky et al. 2008), although there are also several examples where no temperature-size clines have been found (e.g., Adams and Church 2008).

Bergmann's rule has been demonstrated over a wide range of temporal scales. For example, Smith et al. (1995) used fossilized fecal pellets of the bushy-tailed wood rat (Neotoma cinerea) to show that body size had changed with temperature fluctuations during the past 25,000 years, whereas Teplitsky et al. (2008) showed a declining body size over the past 47 years in red-billed gulls (Larus novaehollandie scopulinus).

Despite the many convincing examples of phenotypic trends supporting Bergmann's rule, there is surprisingly little known about the underlying mechanisms giving rise to such temperature-size clines, although they are often interpreted as an evolutionary adaptation to conserve heat loss in cold climates (or dissipate heat in warm climates). Whether these body size clines are caused by an evolutionary adaptation, which assumes that genetic change has taken place, or are due to a phenotypic response (i.e., plasticity) to temperatures is not generally known (Partridge and Coyne 1997). Studies that have addressed the 


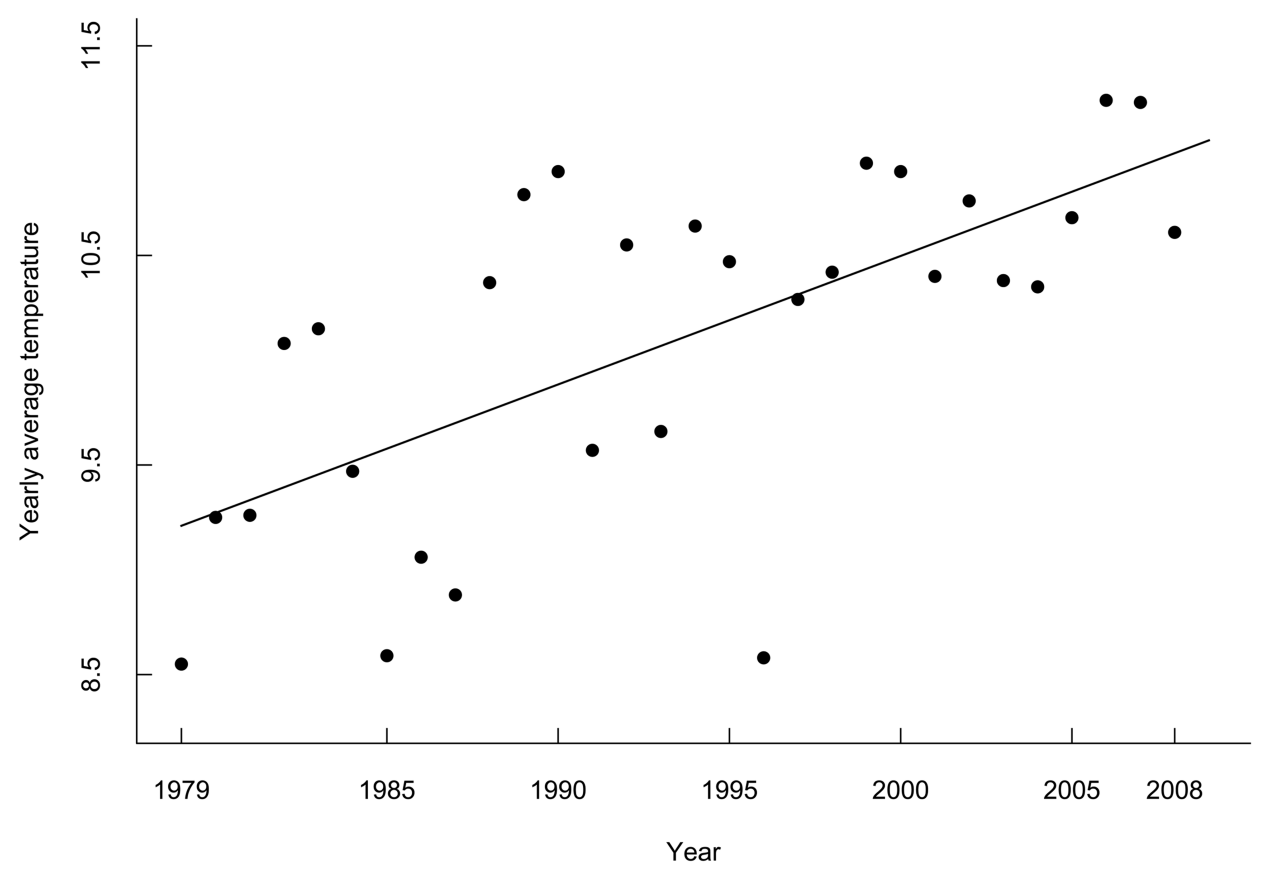

Figure 1: There has been a significant increase in mean annual temperature over the study period $\left(b=0.06, \mathrm{SE}=0.013, t_{28}=4.74\right.$, $P<.001)$.

question of whether size clines are due to environmental or genetic reasons have found support for both cases (Laugen et al. 2005; Teplitsky et al. 2008).

The importance of explicitly considering genetic change is highlighted by some recent empirical studies that have shown that a phenotypic change need not be mirrored at the genetic level (see review by Gienapp et al. 2008) or may even be in the direction opposite the observed genetic change (e.g., Merilä et al. 2001). Clearly, support for evolutionary change must come from demonstrating that a genetic change has taken place. Unfortunately, this is no easy task, and it has, in the past, often required an experimental approach (e.g., Laugen et al. 2005). More recently, the increased application of the animal model (Henderson 1950; Kruuk 2004) in natural populations has opened the possibility that researchers can address this question using longitudinal data within a quantitative genetic setting by examining temporal changes in mean breeding values over time, which can be taken to represent a genetic change (Kruuk 2004; Hadfield et al. 2010), thereby allowing a test of whether phenotypic changes are of genetic origin or due to plasticity.

Bergmann developed his observation only in the context of thermoregulation, and hence Bergmann's rule deals explicitly with body size-temperature clines (Bergmann 1847; Watt et al. 2010). It is important to point out, however, that there are many different selection pressures in- fluencing body size in addition to that of adaptation to external temperatures (Mayr 1956). Factors such as food availability (McAdam and Boutin 2003) and population density (Damuth 1981) are known to lead to changes in body size-related traits (such as body mass or tarsus length) in many species, and changes in environmental factors that covary with but are not causally related to temperature may therefore erroneously give support for Bergmann's rule.

Our goals in this study were to provide insight into the mechanisms behind Bergmann's rule and to explore the temporal phenotypic and genetic patterns in adult body mass and tarsus length in three long-term (1979-2008) study populations of great tit (Parus major) in the Netherlands. According to Bergmann's rule, we predicted that great tits would have become smaller over time because of the recent increase in both yearly mean temperature (fig. 1) and spring temperature (Husby et al. 2010). Thus, we examined first whether adult body mass and tarsus length had changed significantly over the study period and second whether there had been changes in selection pressure over time. However, as there must also be a heritable basis of traits for there to be a response to selection, we next examined the quantitative genetic basis of adult body mass and tarsus length in our populations. We then explicitly tested whether the observed phenotypic changes over time were due to microevolutionary change. Finally, 
we used information from a range of different environmental measures to try to disentangle what environmental factors were most important in driving the observed phenotypic changes.

\section{Material and Methods}

\section{Study Species, Study Area, Field Procedures, and Data}

Great tits are small (14-22 g), insectivorous passerines distributed throughout most of Europe and some parts of Asia (Gosler 1993). The data used in this study were collected from three different populations in The Netherlands-Hoge Veluwe (HV), Oosterhout $(\mathrm{OH})$, and Vlieland (VL) - as part of an ongoing long-term study started in 1955 (van Balen 1973). Systematic collection of adult body mass data, however, started only in the late 1970s, and thus we restricted our analyses to individuals that were caught between 1979 and 2008. For more details about the study populations, see van Balen (1973).

In all areas, nest boxes were visited at least once every week during the breeding season (April-June), and when the young were $7-10$ days old (10-15 on VL), all chicks were ringed, the parents were caught on the nest with a spring trap, and measurements were taken. All adult body mass measurements were taken to the nearest $0.1 \mathrm{~g}$ with a Pesola spring balance, and tarsus length was measured to the nearest $0.1 \mathrm{~mm}$ with a sliding calliper. Parents already ringed were identified, and unringed (immigrant) birds were given a metal ring with a unique number for subsequent identification. For more information about the traits and populations used in this study, see table 1.

Because variation in adult body mass is influenced by a large number of different factors (e.g., time of day when bird was caught, breeding status, age) that could potentially bias our results, we included only measurements of adult breeding birds (i.e., adults caught during chick feeding; see above) that were caught between 0650 and 2100 hours during the breeding season and had not been subject to manipulation. In addition, we corrected body mass for a number of fixed effects to ensure that temporal changes in body mass were not due to, for example, changes in measurement technique over the study period (see table 2).

\section{Population-Level Trends}

Bergmann's rule relates to the overall "size" of an animal, and as in many other studies (Yom-Tov et al. 2006; Teplitsky et al. 2008), we used body mass and tarsus length as proxies for overall body size. Both adult body mass and tarsus length were normally distributed (visual inspection of data, including repeated records). We used linear mixed models to test for temporal change in adult body mass and tarsus length. Individual identity and year were included as random effects in all analyses.

Our goal was to study the within-population temporal changes in adult body mass and tarsus length, and we therefore performed a separate analysis for each population. However, we also included all three populations in one global model and tested for between-population differences in temporal trends.

ASReml-R, version 2.0 (Gilmour et al. 2006), was used to fit all linear mixed models. Statistical significance of fixed effects was assessed from their conditional Wald $F$ test statistics to respect principles of marginality. Because there seemed to be substantial nonlinear changes in body mass and tarsus length over time (see figs. 2,3) and such nonlinearity has been documented in other cases (Salewski et al. 2010), we also tested for nonlinearity in the temporal trends, using generalized additive models implemented in the mgcv library (Wood 2006) in R 2.8.1 (R Development Core Team 2007), using year as the smoothing parameter (see Salewski et al. 2010).

\section{Selection Analysis}

We estimated fecundity selection on each trait in each population separately, using the yearly number of offspring that an individual recruited to the breeding population ("recruited" means that an offspring was recorded as a breeding bird in the population in years after its year of birth) as an estimate of fitness, as it is only offspring that recruit to the breeding population that contribute to any

Table 1: Mean values, standard deviations, and sample sizes for the different traits in each population of great tits

\begin{tabular}{lccccccrr}
\hline & \multicolumn{3}{c}{ Adult body mass } & & \multicolumn{3}{c}{ Tarsus length } \\
\cline { 2 - 4 } & \multicolumn{2}{c}{ HV } & OH & VL & & HV & OH & VL \\
\hline Trait mean (SD) & $17.64(.83)$ & $17.73(.86)$ & $17.47(.87)$ & & $19.77(.63)$ & $19.68(.71)$ & $19.46(.69)$ \\
Individuals & 3,308 & 975 & 3,026 & & 3,283 & 966 & 3,191 \\
Records & 5,659 & 1,741 & 5,309 & & 5,512 & 1,598 & 5,520 \\
\hline
\end{tabular}

Note: $\mathrm{HV}=$ Hoge Veluwe, $\mathrm{OH}=$ Oosterhout, and $\mathrm{VL}=$ Vlieland. Adult body mass measurements were restricted to the criteria outlined in "Material and Methods," and means include repeated records. 
Table 2: ANOVA table from the linear-mixed-model analysis of adult body mass $(A)$ and tarsus length $(B)$ for the three study populations

\begin{tabular}{|c|c|c|c|c|c|c|}
\hline & \multicolumn{2}{|c|}{$\mathrm{HV}$} & \multicolumn{2}{|c|}{$\mathrm{OH}$} & \multicolumn{2}{|c|}{$\mathrm{VL}$} \\
\hline & df & $F$ & df & $F$ & df & $F$ \\
\hline \multicolumn{7}{|l|}{ A. Body mass: } \\
\hline Year & $1,133.8$ & $10.58^{\star *}$ & $1,1,706.7$ & $3.90^{*}$ & $1,396.2$ & $9.49^{\star *}$ \\
\hline Sex & $1,3,253.7$ & $199.90^{* * *}$ & $1,943.5$ & $92.68^{\star * *}$ & $1,2,988.2$ & $422.4^{\star \star *}$ \\
\hline Catching date (April date) & $1,3,068.2$ & $81.16^{\star * *}$ & $1,1,109.6$ & $11.56^{\star * *}$ & $1,3,146.0$ & $200.7^{\star * *}$ \\
\hline Age of chicks & $1,3,566.3$ & $36.69^{* * *}$ & $1,1,279.8$ & $5.059^{*}$ & $1,3,546.9$ & $25.92^{* * *}$ \\
\hline Age of individual & $1,3,401.5$ & $68.07^{\star * *}$ & $1,1,235.0$ & $25.84^{* * *}$ & $1,3,486.8$ & $72.92^{\star * *}$ \\
\hline Time of day when captured & $1,3,925.2$ & $247.70^{* * *}$ & $1,1,320.6$ & $80.84^{* * *}$ & $1,3,702.5$ & $308.3^{\star * *}$ \\
\hline Observer identity & $36,3,285.4$ & $3.387^{* * *}$ & $24,1,423.7$ & $4.02^{* * *}$ & $40,2,600.0$ & $3.41^{\star * *}$ \\
\hline Year $\times$ sex & $1,3,325.1$ & 2.00 & $1,935.6$ & 4.51 & $1,2,988.6$ & 2.65 \\
\hline \multicolumn{7}{|l|}{ B. Tarsus length: } \\
\hline Year & $1,92.4$ & $3.36^{\dagger}$ & $1,194.2$ & .05 & $1,123.2$ & $7.52^{\star \star}$ \\
\hline Sex & $1,3,267.5$ & $757.90^{* * *}$ & $1,814.2$ & $307.50^{* * *}$ & $1,3,168.3$ & $866.20^{\star * *}$ \\
\hline Observer identity & $35,2,285.3$ & $5.64^{\star * *}$ & $25,537.6$ & $8.62^{\star * *}$ & $39,408.1$ & $22.71^{\star * *}$ \\
\hline Age of individual & $1,330.1$ & .09 & $1,780.7$ & $6.52^{\star}$ & $1,3,423.1$ & 1.89 \\
\hline Year $\times$ sex & $1,3,693.1$ & .47 & $1,815.2$ & .67 & $1,3,557.1$ & .01 \\
\hline
\end{tabular}

selection response. Similarly, viability selection was estimated with local survival defined as 1 if the individual survived to the next breeding season and 0 if it did not survive. For each population, we conducted year-specific analysis (excluding 2008 because data for the 2009 season were not available at the time of analysis) by standardizing each trait (adult body mass and tarsus length) to have zero mean and unit variance (thus creating $z$ scores) within each year and population. In the fecundity selection analyses, yearly fitness values (number of recruits) were divided by the mean number of recruits produced in the given year and population to give relative fitness scores $(\omega)$ for each individual. For the viability selection analyses, we used the survival information directly.

Standardized selection gradients $(\beta)$ for adult body mass and tarsus length were measured with a least squares regression technique as the regression slope between relative fitness and standardized trait values (Lande and Arnold 1983). Because relative fitness $(\omega)$ and survival do not follow a Gaussian distribution, inferences from a least squares regression will be unreliable, and we therefore assessed statistical significance of the standardized selection gradients with a generalized linear mixed model (GLMM; log link function for fecundity selection analyses and binomial link function for viability selection analyses), including individual identities as random effects to account for repeated measurements on individuals. Yearly standardized selection gradients and their standard errors and significance levels (as estimated from the GLMM analysis) are available at Dryad (http://dx.doi.org/10.5061/dryad .8989: tables A1 and A2 for fecundity selection analysis of adult body mass and tarsus length, respectively, and tables A3 and A4 for viability selection analysis of adult body mass and tarsus length, respectively).

We also tested for temporal change in selection pressure by regressing annual standardized selection gradients against year with a least squares regression. The least squares regression analyses were performed in R 2.8.1 and the GLMM analysis in ASReml-R (Gilmour et al. 2006). All tests were two-tailed, with a significance threshold of $P=.05$.

\section{Pedigree}

We reconstructed a pedigree based on social information (i.e., from field observations), and so the pedigree can contain errors through the paternal line (due to extrapair paternity [EPP]). Although the rate of EPP is unknown in the $\mathrm{HV}$ and $\mathrm{OH}$ populations, it has been estimated to be as low as 3.5\% extrapair young in the VL population (excluding one nest in which all offspring were sired by an extrapair male; Verboven and Mateman 1997). Furthermore, there is no reason to expect the EPP levels to be higher in the $\mathrm{HV}$ and $\mathrm{OH}$ populations, because it is also generally low in other populations of great tits (e.g., Lubjuhn et al. 1999). At such low levels, EPP has only a 


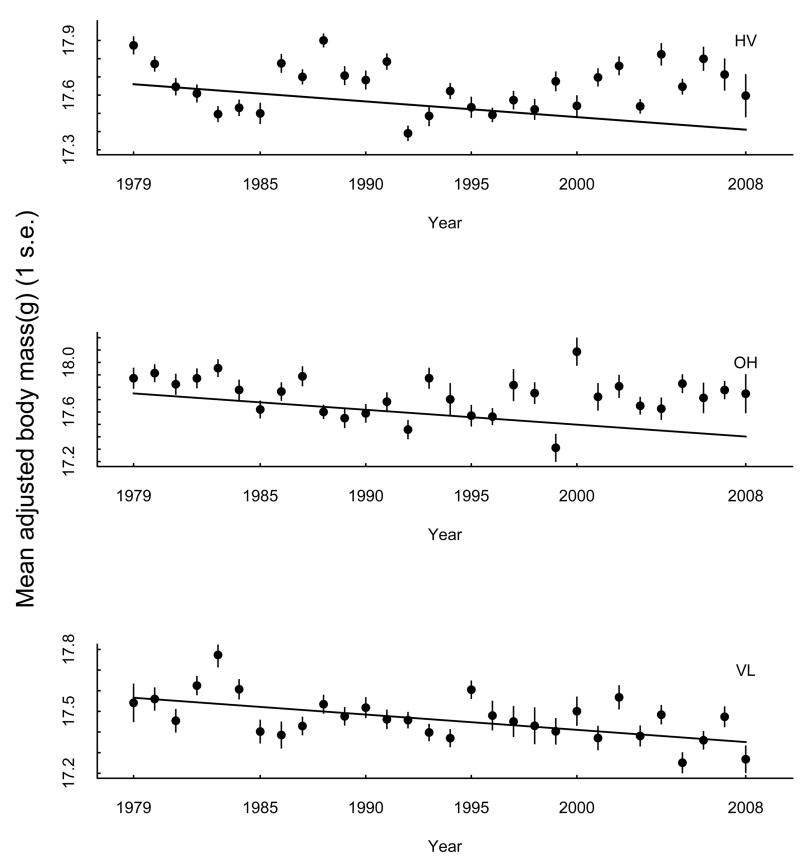

Figure 2: Temporal patterns in yearly average adult body mass (corrected for time of measurement) in the three study populations $(\mathrm{HV}=$ Hoge Veluwe, $\mathrm{OH}=$ Oosterhout, and VL = Vlieland $)$. All trends were statistically significant; see table 2, part A, for details.

negligible effect on the estimated additive genetic variance component, and thus on heritability estimates, when samples are as large as those in this study (Charmantier and Reale 2005). Some chicks were cross-fostered, in which case we used the genetic parents rather than the social parents in the pedigree. To preserve sibship information and maximize pedigree information, we dummy-coded parents whenever information about either the male or the female was missing. Note that because of the very low EPP rate, this is very unlikely to create any bias.

The pedigree for the HV population had a total of 3,460 individuals, with a mean relatedness of $4.45 \times 10^{-4}, 1,139$ maternities, 1,125 paternities, 609 full siblings, $247 \mathrm{ma}-$ ternal half-sibs, and 232 paternal half-sibs, and it spanned a maximum of 16 generations. The $\mathrm{OH}$ population pedigree had a total of 1,062 individuals, with a mean relatedness of $4.5 \times 10^{-3}, 589$ maternities, 559 paternities, 385 full siblings, 307 maternal half-sibs, and 156 paternal halfsibs, and it spanned a maximum of 18 generations. Finally, the pedigree of the VL population had a total of 3,686 individuals, with a mean relatedness of $7.54 \times 10^{-3}, 2,661$ maternities, 2,615 paternities, 2,077 full siblings, 1,208 maternal half-sibs, and 1,073 paternal half-sibs, and it spanned a maximum of 40 generations. Pedigree statistics were obtained with the $\mathrm{R}$ package pedantics (Morrissey and Wilson 2010).

\section{Quantitative Genetic Analyses}

To separate environmental and genetic sources of variation in body mass and tarsus length, we used a residual maximum likelihood mixed model ("animal model"). Animal models use information about pairwise relatedness between all individuals in the population to partition the phenotypic trait variance into its additive genetic variance component and its environmental (and other nongenetic) variance components (Henderson 1950; Lynch and Walsh 1998; Kruuk 2004).

Because we have repeated measures on the same individuals in different environments, we also estimated the permanent environment effect, that is, the within-individual variance associated with environmental effects (or nonadditive effects such as dominance or epistasis; Kruuk 2004). Year was included as a random effect to account for temporal heterogeneity in environmental effects on the phenotypes. Note that year was also included as a covariate in all animal-model analyses to avoid bias in the breeding

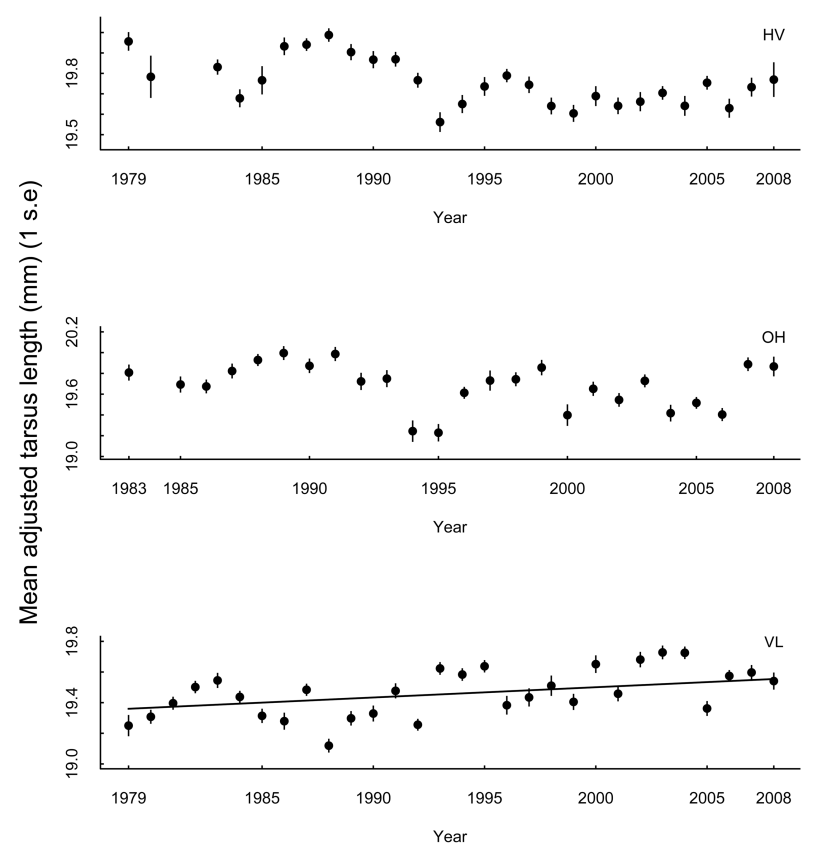

Figure 3: Change in yearly average tarsus length in the three study populations $(\mathrm{HV}=$ Hoge Veluwe, $\mathrm{OH}=$ Oosterhout, and $\mathrm{VL}=$ Vlieland) over time. Only the trend for VL is statistically significant and so is shown as a line here; see table 2, part B, for details. Note that in some years data were not available; see Dryad (http:// dx.doi.org/10.5061/dryad.8989) for detailed information about yearly sample size. 
values toward the phenotypic trend (Postma 2006). Traitspecific fixed effects that were significant in the phenotypic analysis were included in the animal-model analyses (see table 2).

Thus, after correction for the fixed effects mentioned above, our animal models partitioned the phenotypic variance $\left(V_{\mathrm{P}}\right)$ in adult body mass and tarsus length into four components:

$$
V_{\mathrm{P}}=V_{\mathrm{A}}+V_{\mathrm{PE}}+V_{\text {Year }}+V_{\mathrm{R}} \text {, }
$$

where $V_{\mathrm{A}}$ is the additive genetic variance, $V_{\mathrm{PE}}$ is the permanent environmental variance, $V_{\text {Year }}$ is the variance associated with the year (common environment variance), and $V_{\mathrm{R}}$ is the residual variance. Heritability of the two traits was then calculated as $h^{2}=V_{\mathrm{A}} / V_{\mathrm{P}}$ (Falconer and Mackay 1996). We also report the coefficient of additive genetic variance $\left(\mathrm{CV}_{\mathrm{A}}=100\left(V_{\mathrm{A}}\right)^{1 / 2} /\right.$ trait mean $)$ as a measure for comparison between populations and with other studies (Houle 1992).

To examine whether there had been a genetic change in population composition over time, we assessed the significance of the temporal change in breeding values from the posterior distribution of change in breeding values over time, using a Bayesian Markov chain Monte Carlo (MCMC) animal-model approach (Hadfield et al. 2010). We included the same fixed effects and random effects as described under the animal-model analysis, and we used weakly informative priors set to one-fourth of the phenotypic variance. The models were run for a minimum of 40,000 iterations, with a burn-in phase of 10,000 iterations. Mixing of the chains was assessed manually by visual inspection, and the number of iterations was increased to achieve proper mixing.

All animal models were run in the software ASReml, version 2.0 (Gilmour et al. 2006). The Bayesian MCMC animal models were run in $\mathrm{R}$ with the MCMCglmm package (Hadfield 2010).

\section{Environmental Variables}

Because many environmental factors may contribute to the selection pressure on body size (Mayr 1956), we collected information about a range of different environmental variables known to be important biological factors in our populations: beech crop index (Perdeck et al. 2000), date of the caterpillar peak (Visser et al. 2006), synchrony between the date of the caterpillar peak and the mean laying date (Visser et al. 2006), temperature during February-March as a proxy for winter severity (Perdeck et al. 2000), yearly mean temperature, and finally, population density (measured as number of breeding pairs). Unfortunately, information on beech crop index, date of caterpillar peak, and synchrony (the difference between the date of the caterpillar peak and the yearly mean laying date) was available only for the HV population from 1985 onward (see Visser et al. 2006; Husby et al. 2009), and thus we had to restrict our analysis to the HV population and the years 1985-2008. We included adult body mass (and tarsus length) as response variables in a linear mixed model, with the above environmental factors included as explanatory variables and individual identity as a random effect to account for repeated measures on the same individuals. All environmental variables were mean centered before analysis, and statistical significance was assessed by the conditional Wald test statistics in ASReml-R (Gilmour et al. 2006).

\section{Results \\ Population-Level Trends}

Adult body mass declined significantly in all three populations $\left(b_{\mathrm{hv}}=-0.013 \pm 0.004 \mathrm{~g} /\right.$ year, $b_{\mathrm{oh}}=-0.012 \pm$ $0.006 \mathrm{~g} /$ year, and $b_{\mathrm{vl}}=-0.015 \pm 0.005 \mathrm{~g} /$ year; see table 2, pt. A; fig. 2) over the study period. There were strong effects of the time of day when a bird was measured, the day of capture, age of the chicks at the time of capture, observer identity, and sex (table 2, pt. A). Because of the very similar negative trend in all three populations, there was no significant population $\times$ year interaction when all populations were tested simultaneously in the same model $\left(F_{2,11,345.8}=0.013, P=.98\right)$.

Tarsus length did not change significantly over time in the $\mathrm{HV}$ and $\mathrm{OH}$ populations $\left(b_{\mathrm{hv}}=-0.007 \pm 0.004 \mathrm{~mm} /\right.$ year and $b_{\text {oh }}=-0.006 \pm 0.006 \mathrm{~mm} /$ year; table 2, pt. B; fig. 3), but it showed a significant increase in the VL population $\left(b_{\mathrm{v} 1}=0.0126 \pm 0.004 \mathrm{~mm} /\right.$ year; table 2 , pt. B; fig. $3)$. The between-populations difference in temporal patterns was clearly indicated by a highly significant population $\times$ year interaction when all populations were tested in the same model $\left(F_{2,7,992.0}=42.94, P<.001\right)$.

As evident from figures 2 and 3 , there was a great deal of nonlinearity in the temporal phenotypic trends in body mass and tarsus length. This nonlinearity was confirmed by additive models; there were significant nonlinear effects of year on body mass (HV: $n=5,659, F=12.99, P<$ .001 ; OH: $n=1,741, F=9.05, P<.001$; VL: $n=5,309$, $F=3.02, P=.0014$ ) and tarsus length (HV: $n=5,512$, $F=18.73, \quad P<.001 ;$ OH: $n=1,598, \quad F=11.54, \quad P<$ .001 ; VL: $n=5,520, F=18.64, P<.001)$ in all populations.

\section{Fecundity Selection Analyses: Adult Body Mass}

Across the study period, there was overall positive directional selection on adult body mass in the $\operatorname{HV}(\beta=$ 
Table 3: Components of phenotypic variance in adult body mass and tarsus length and their standard errors, as estimated from an animal model

\begin{tabular}{lccccccc}
\hline & \multicolumn{3}{c}{ Adult body mass (SE) } & & \multicolumn{3}{c}{ Tarsus length (SE) } \\
\cline { 2 - 3 } \cline { 7 - 8 } & HV & OH & VL & & HV & OH & VL \\
\hline$V_{\mathrm{A}}$ & $.312(.025)$ & $.303(.043)$ & $.270(.023)$ & & $.168(.014)$ & $.121(.024)$ & $.174(.020)$ \\
$V_{\mathrm{PE}}$ & $.130(.021)$ & $.104(.032)$ & $.144(.017)$ & & $.086(.012)$ & $.108(.020)$ & $.017(.015)$ \\
$V_{\mathrm{Y} e a r}$ & $.014(.004)$ & $.011(.007)$ & $.004(.002)$ & & $.005(.002)$ & $.044(.017)$ & $.080(.024)$ \\
$V_{\mathrm{R}}$ & $.186(.005)$ & $.259(.012)$ & $.209(.006)$ & & $.062(.002)$ & $.109(.006)$ & $.540(.013)$ \\
$V_{\mathrm{P}}$ & $.642(.015)$ & $.678(.028)$ & $.627(.016)$ & & $.320(.008)$ & $.381(.023)$ & $.811(.029)$ \\
$\mathrm{CV}_{\mathrm{A}}$ & 3.167 & 3.105 & 2.974 & & 2.073 & 1.768 & 2.144 \\
$h^{2}$ & $.486(.034)$ & $.447(.053)$ & $.431(.031)$ & $.525(.039)$ & $.317(.058)$ & $.214(.023)$ \\
\hline
\end{tabular}

Note: $\mathrm{HV}=$ Hoge Veluwe, $\mathrm{OH}=$ Oosterhout, and $\mathrm{VL}=$ Vlieland. $V_{\mathrm{P}}=$ phenotypic variance, $V_{\mathrm{A}}=$ additive genetic variance, $V_{\mathrm{PE}}=$ permanent environment variance, $V_{\text {Year }}=$ year variance, $V_{\mathrm{R}}=$ residual variance, $\mathrm{CV}_{\mathrm{A}}=$ coefficient of additive genetic variance, and $h^{2}=$ heritability.

$\left.0.115, \mathrm{SE}=0.025, \chi_{1}^{2}=23.65, P<.001\right)$ and $\mathrm{OH}(\beta=$ $\left.0.042, \mathrm{SE}=0.037, \chi_{1}^{2}=6.47, P=.011\right)$ populations but not in the VL population $\left(\chi_{1}^{2}=0.05, P=.832\right)$. However, year-specific analyses indicated that selection on adult body mass was not very consistent across years, with only seven significant selection gradients (all positive; table A1) and large interannual variation in the strength and sign in the three populations (see table A1). Also, the strength of the selection gradients changed significantly over the study period in the $\mathrm{OH}$ population $(b=0.012, \mathrm{SE}=$ $0.005, P=.02)$ but not in the HV $(b=-0.007, \mathrm{SE}=$ $0.004, P=.069)$ and VL $(b=0.004, \mathrm{SE}=0.002, P=$ .089) populations.

\section{Fecundity Selection Analyses: Adult Tarsus Length}

Across all years, we found significant directional selection in the $\mathrm{HV}$ population $\left(\beta=0.055, \mathrm{SE}=0.026, \chi_{1}^{2}=\right.$ 4.11, $P=.043)$ but not in the $\mathrm{OH}\left(\chi_{1}^{2}=3.67, P=\right.$ $.056)$ and VL $\left(\chi_{1}^{2}=0.093, P=.76\right)$ populations. Again, the year-specific analyses showed large interannual variation in the direction and strength of selection (table A2). We did not find any indication that selection on tarsus length had changed over the study period in any of the three populations $(P=.98, .44$, and .42 for the $\mathrm{HV}, \mathrm{OH}$, and VL populations, respectively).

\section{Viability Selection Analyses: Adult Body Mass}

Across all years, we found significant positive directional selection on body mass in the $\mathrm{OH}(\beta=0.025, \mathrm{SE}=$ $\left.0.011, \chi_{1}^{2}=4.05, P=.044\right)$ and $\mathrm{VL}(\beta=0.021, \mathrm{SE}=$ 0.007, $\left.\chi_{1}^{2}=6.73, P=.009\right)$ populations but not in the $\mathrm{HV}$ population $\left(\chi_{1}^{2}=0.04, P=.84\right)$. Year-specific analyses indicated little consistency in the direction of selection both within the same population between years and across populations in the same year (see table A3). There was no temporal change in selection pressures over time in any of the three populations $(P=.54, .64$, and .49 for $\mathrm{HV}$, $\mathrm{OH}$, and VL, respectively).

\section{Viability Selection Analyses: Adult Tarsus Length}

Tarsus length was under positive directional selection in the $\operatorname{HV}\left(\beta=0.027, \mathrm{SE}=0.007, \chi_{1}^{2}=14.86, P<.001\right)$ and VL $\left(\beta=0.015, \mathrm{SE}=0.007, \chi_{1}^{2}=5.01, P=.025\right)$ populations but not in the $\mathrm{OH}$ population $\left(\chi_{1}^{2}=0.003\right.$, $P=.96)$, when analyzed across all years. Yet both the strength and the direction of selection fluctuated between years within the same population and across populations within the same year (table A4). Again, there was no support for a temporal change in selection patterns in any of the populations $(P=.16, .84$, and .21 for $\mathrm{HV}, \mathrm{OH}$, and $\mathrm{VL}$, respectively).

\section{Quantitative Genetic Analyses: Estimation of Heritability}

Heritability of both adult body mass and tarsus length was significantly greater than 0 in all three populations (table 3; for adult body mass: HV: $t_{6,016}=14.29$, OH: $t_{1,940}=$ 8.43, and VL: $t_{5,350}=13.90$, with all $P<.0001$; for tarsus length: $\mathrm{HV}: t_{5,511}=13.46, \mathrm{OH}: t_{1,597}=5.47$, and VL: $t_{5,519}=9.30$, with all $P<.0001$ ), and heritability of body mass did not differ significantly between populations (HV/ $\mathrm{OH}: t_{7,956}=0.62, P=.54 ; \mathrm{HV} / \mathrm{VL}: t_{11,366}=1.19, P=$ .23; OH/VL: $t_{7,290}=0.26, P=.79$ ). Heritability of tarsus length differed significantly between $\mathrm{HV}$ and $\mathrm{OH}$ $\left(t_{7,108}=2.98, P=.003\right)$ and between $\mathrm{HV}$ and $\mathrm{VL}$ $\left(t_{11,030}=6.87, P<.001\right)$ but not between $\mathrm{OH}$ and $\mathrm{VL}$ $\left(t_{7,116}=1.65, P=.10\right)$. The significant difference in heritability of tarsus length between populations was mainly due to differences in residual variance, as both $V_{\mathrm{A}}$ estimates and $\mathrm{CV}_{\mathrm{A}}$ estimates indicated little between-population variation (table 3 ). 
Quantitative Genetic Analyses: Testing for Microevolution

The observed phenotypic decline in adult body mass in the three populations and the increase in tarsus length in VL could be either due to a genetic change or a result of phenotypic plasticity. We therefore tested explicitly for genetic change by using Bayesian animal models and assessing the posterior distribution of the change in breeding values over time (Hadfield et al. 2010). There was no indication that the breeding values for adult body mass or tarsus length changed significantly over the study period in any of the three study populations (see table 4), and the sign of the genetic change was, furthermore, the opposite of that of the phenotypic change. Hence, the observed phenotypic changes were not due to evolutionary change but rather were a result of a plastic adjustment to changing environmental conditions.

\section{Exploring Environmental Variables Causing the Phenotypic Decline}

Plastic adjustment of body mass (and tarsus length in VL) could be in response to increased temperature but could also be in response to other environmental factors. We therefore explored how variation in adult body mass and tarsus length was influenced by a number of different environmental factors. We found that, after model reduction, density had a negative effect on adult body mass $(b=$ $\left.-0.071, \mathrm{SE}=0.0014, F_{1,1,229.4}=25.64, P<.001\right)$, whereas synchrony, caterpillar peak date, and beech crop index were all positively related to adult body mass $(b=$ $0.077, \mathrm{SE}=0.022, F_{1,1,229.4}=25.64, P<.001 ; b=0.121$, $\mathrm{SE}=0.026, \quad F_{1,732.5}=12.69, \quad P<.001 ;$ and $b=0.073$, $\mathrm{SE}=0.015, F_{1,1,087.7}=24.65, P<.001$ for synchrony, caterpillar peak date, and beech crop index, respectively). Temperature during the period February-March and yearly mean temperatures, however, were not significant $\left(F_{1,1,187.6}=0.88, \quad P=.35\right.$ for February-March and $F_{1,3,836.2}=3.46, P=.063$ for yearly mean values). Because only caterpillar peak date $\left(F_{1,22}=22.62, \quad b=-0.87\right.$, $\mathrm{SE}=0.18, P<.001)$ and synchrony $\left(F_{1,22}=9.32, b=\right.$
$0.59, \mathrm{SE}=0.19, P=.006)$ changed significantly over the examined period (1985-2008), the decline in adult body mass is most likely due to a change in food conditions and the associated increase in mistiming (see "Discussion"). We found that none of the environmental variables examined had an effect on tarsus length (all $P>.14$ ).

\section{Discussion}

Few studies have examined whether temporal changes in body size in relation to increased temperatures (Bergmann's rule) are of genetic or environmental origin or both. Here we have provided a detailed analysis of the causes of phenotypic changes in adult body mass and tarsus length in three long-term study populations of great tits in relation to the recent increase in temperature (fig. 1). In common with many other recent studies examining the effect of increased temperatures on body size (Millien 2004; but see Yom-Tov et al. 2006; Teplitsky et al. 2008; Salewski et al. 2010), we found that adult body mass declined over the study period (fig. 2). Contrary to most studies examining Bergmann's rule, we disentangled the phenotypic trend and showed that this decline was not due to microevolutionary change but was a result of phenotypic adjustment (plasticity). This plasticity could be in response to the increase in temperatures or to other environmental factors. Interestingly, we did not find that temperature had a strong influence on body mass; instead, increased mistiming between the peak in food abundance and the chick-rearing period was the more important factor driving the decline in adult body mass. Although our data are correlational and thus unable to separate cause and effect, our findings suggests that changes in food abundance are more important than temperature in explaining the observed size cline in these populations.

\section{Temporal Changes in Body Size Traits}

Adult body mass declined significantly in all three populations over the 30 -year period, and our results are thus in line with those of other studies that have found de-

Table 4: Bayesian Markov chain Monte Carlo animal-model analyses assessing significance of temporal trends in estimated breeding values for adult body mass and tarsus length

\begin{tabular}{lccccccc}
\hline & \multicolumn{3}{c}{ Adult body mass } & & \multicolumn{3}{c}{ Tarsus length } \\
\cline { 2 - 3 } & $\mathrm{HV}$ & $\mathrm{OH}$ & $\mathrm{VL}$ & & $\mathrm{HV}$ & $\mathrm{OH}$ & $\mathrm{VL}$ \\
\hline $\begin{array}{l}\text { Year: } \\
\beta\end{array}$ & .0006 & .0042 & .0019 & & .0007 & .0021 & .0024 \\
$95 \% \mathrm{CI}$ & $-.0024, .0038$ & $-.0014, .0113$ & $-.0023, .0065$ & $-.0014, .0028$ & $-.0024, .0065$ & $-.0007, .0054$ \\
\hline
\end{tabular}

Note: $\mathrm{HV}=$ Hoge Veluwe, $\mathrm{OH}=$ Oosterhout, and $\mathrm{VL}=$ Vlieland. The estimate of the mean $(\beta)$ of the posterior distribution of estimated genetic change and its $95 \%$ confidence interval (CI) are given. There was no indication of a significant genetic change over time in any of the traits for the three populations. 
clining adult body mass during periods of increased temperatures (e.g., Smith et al. 1995; Teplitsky et al. 2008). Two other studies of great tits have also reported a temporal decline in adult body mass, with decreases of 0.036 g/year over the period 1968-2002 (Yom-Tov et al. 2006) and 0.023 residual g/year (Cresswell et al. 2009). In comparison, the decrease in adult body mass found here was between 0.013 and $0.015 \mathrm{~g} /$ year. Although these figures are not easily comparable because different approaches were used to estimate the trends, they nevertheless illustrate the range of the rates of decline found. Also other, closely related species and species similar in size to the great tit, such as bullfinches (Pyhrulla pyhrulla), blue tits (Cyanistes caeruleus), and dunnocks (Prunella modularis) in Britain, show a decline of $\sim 0.014$ (residual) g/year between 1968 and 2003 (Yom-Tov et al. 2006). As shown by Salewski et al. (2010), temporal changes in body size can show complex nonlinear patterns, and our additive models also confirmed this in our study populations for both body mass and tarsus length. Despite the large interannual variation in body size, there were still significant linear declines in body mass in all three populations, however; thus, overall, great tits have become smaller over the study period even though size fluctuated considerably.

For tarsus length, we observed an increasing size over time in the VL population, something that can be interpreted to support Allen's rule, which predicts that homeothermic animals in colder environments will have relatively smaller appendages than those in warm environments (Scholander 1955). In contrast to Allen's rule, however, other studies have noted a decline in tarsus length with increasing temperatures (Yom-Tov 2001; Teplitsky et al. 2008).

\section{Selection Analysis}

Characterizing patterns of selection on body size can provide important insights into the likelihood that "Bergmann clines" arise as a result of microevolutionary change, since, given a heritable basis of a trait, selection must act on the trait to produce a response. An evolutionary change is unlikely if there is no selection on the traits.

Although body size-related traits have been found to be under strong directional selection in many studies on birds (Kruuk et al. 2001), mammals (Milner et al. 1999), and other taxa (Kingsolver et al. 2001), there are also abundant examples where morphological traits are under no apparent selection (e.g., Schluter and Smith 1986; Jensen et al. 2004). Selection thus must be measured in each case and not inferred from other studies. Here we found that there was, across all years, only weak positive fecundity selection on adult body mass in two populations (HV and $\mathrm{OH}$; see table $\mathrm{A} 1$ ) and positive viability selection in the
$\mathrm{OH}$ and VL populations (table A3). Tarsus length was also under weak positive directional fecundity selection in the HV but not the $\mathrm{OH}$ and VL populations (table A2) and was under positive directional viability selection in the HV and VL but not the $\mathrm{OH}$ population.

These selection analyses highlight two things: first, selection was weak and inconsistent between years (tables A1-A4), and second, the direction of selection was opposite that of the phenotypic trends. This argues against the observed decline in body mass being a result of microevolutionary change.

Interestingly, there was some indication that selection on body mass had changed over the study period, with increasingly strong selection on body mass in the $\mathrm{OH}$ and VL populations but relaxed selection in the HV population. The temporal increase in strength of selection in the $\mathrm{OH}$ and VL populations contrasts with the observed phenotypic decline in body mass, and at present we do not have a good understanding as to why selection changed over the study period, although changes in the food dynamics seem a likely explanation (see below).

\section{Heritability of Body Size Traits}

The genetic basis of body size-related traits are well studied; heritability of morphological traits is generally moderate to high (for an avian review, see Merilä and Sheldon 2001), and our results also fall within this range. Heritability of adult body mass varied from 0.43 to 0.49 (table 3 ) in our three study populations, and similarly, the heritability of tarsus length varied from 0.22 to 0.53 (table 3 ).

Heritability estimates are often found to differ between populations of the same species, because of both genetic and environmental differences, and combined with different selection pressures in the different populations, heritability could help explain interpopulation variation in declines in body size. For example, in our populations, heritability of tarsus length was significantly different between areas (between $\mathrm{HV}$ and $\mathrm{OH}$ and between $\mathrm{HV}$ and VL; table 3), but in contrast, there were no significant population differences in heritability of adult body mass (table 3).

\section{Microevolutionary Change}

Testing for microevolutionary change in longitudinal studies has generally been carried out with least squares linear regressions to regress mean annual estimated breeding values on year (e.g., Merilä et al. 2001). However, it has recently been suggested that this approach is anticonservative because many of the assumptions of a linear regression are violated when predicted breeding values are used. This is because breeding values are not independent and because there is also a positive correlation across mean 
cohort breeding values (see Hadfield et al. 2010 for details). We therefore used the newly proposed alternative Bayesian animal-model framework implemented by Hadfield et al. (2010) to test for microevolutionary trends. These analyses showed no indication of a significant genetic change over time for adult body mass or tarsus length in any of our three study populations (table 4). This lack of genetic change is perhaps not unexpected, given the inconsistent direction of selection acting on adult body mass and tarsus length over the study period (tables A1-A4). As the Bayesian methodology is a very recent approach, we also tested for microevolutionary change with the linear model approach using best linear unbiased predictions. In line with the findings of Hadfield et al. (2010), these analyses showed a pattern contrasting with that found by the Bayesian approach, with significant genetic change for adult body mass in both the HV and VL populations (A. Husby, unpublished results). Using the "old" approach, we would thus have erroneously concluded that genetic change had taken place in these populations. We caution, however, that the Bayesian approach to estimate genetic change has not been validated with simulated data, and until this is done it is unclear to what extent and degree the Bayesian framework gives correct significance level. The lack of support for microevolutionary change indicates that the observed temporal changes in body mass and tarsus length (in the VL population) are due to a phenotypic adjustment to changes in the environment.

\section{Exploring Environmental Variables Influencing Body Mass and Tarsus Length}

Temperature is the selective agent responsible for the body size clines underlying Bergmann's rule (Stillwell et al. 2007), but it is unlikely to be the only explanation for Bergmann clines, as they have also been demonstrated in ectotherms, such as insects (Stillwell et al. 2007), where the heat conservation argument does not hold. For example, in the seed-feeding beetle (Stator limbatus), body size clines were more related to variation in host plant seed size, moisture, and seasonality than to temperature variation (Stillwell et al. 2007).

We also considered multiple environmental variables and did not find that temperature measured during the most severe winter months or annual mean temperature were significantly related to the decline in body mass. Density, time of the caterpillar peak, and the timing between laying date and caterpillar peak were instead stronger predictors of variation in adult body mass during the breeding season than were the two temperature measures. However, because density has not changed over the study period, this cannot explain the decline in adult body mass. This suggests, therefore, that adults have adjusted their body mass in relation to changes in food abundance during the breeding season (fig. 4). Alternatively, if fledgling body mass has declined over time, as has been found in a different population of great tits (Garant et al. 2004), there could be carryover effects to the adult stage. In our study populations, annual mean fledgling mass did not change in the HV and VL populations during the period 1979$2008(P=.39$ and 1.00 for HV and VL, respectively), but it has declined in the $\mathrm{OH}$ population $(b=-0.042$, $\left.\mathrm{SE}=0.015, \chi_{1}^{2}=6.90, P=.009\right)$. It is therefore likely that the observed temporal decline in adult body mass in the $\mathrm{OH}$ population is due to a carryover effect of reduced fledgling mass caused by the increasing distance between timing of reproduction and the peak in food abundance (Visser et al. 1998). Previous work has shown that fledgling mass declines strongly as mistiming increases (Visser et al. 2006). This suggests that the decline in body mass in the $\mathrm{OH}$ population is not a result of direct adjustment to temperature, even if temperature is an important indirect factor causing the increase in mistiming.
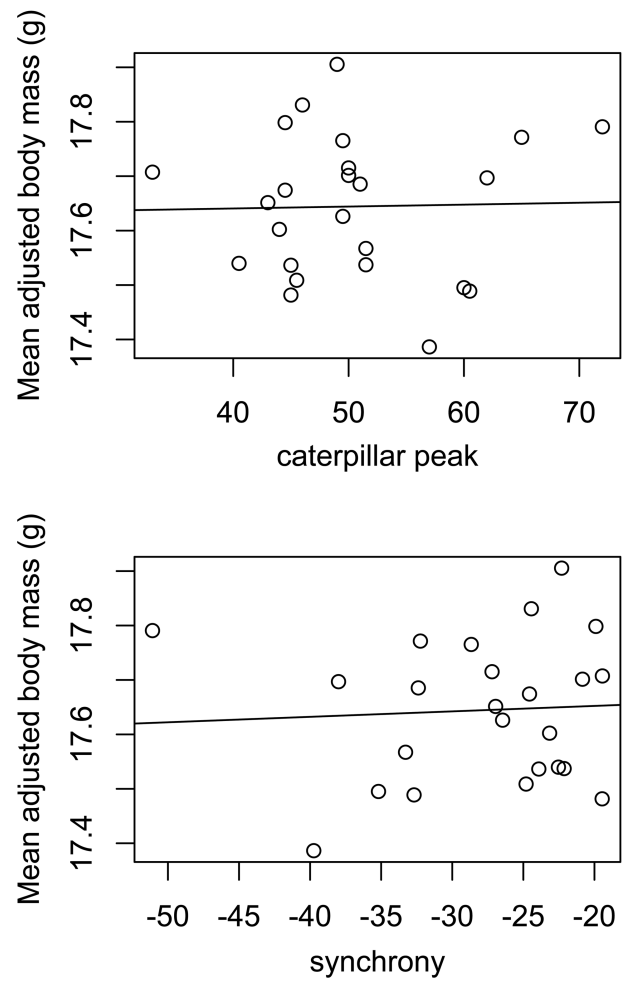

Figure 4: Body mass (corrected for time of measurement) in the Hoge Veluwe population in relation to the peak date in caterpillar abundance (top) and the synchrony between laying date and caterpillar abundance (bottom). Note that the fitted slopes are from a linear regression of annual mean values against annual mean caterpillar abundance and synchrony and that they do not reflect the slopes given from the linear-mixed-model analyses in "Results." 
For the HV and VL populations, however, the decline in adult body mass does not seem to be due to a carryover effect of decreasing mass at the fledgling stage but instead must be due to a change at the adult stage, presumably during the breeding season. To examine this in greater detail, we used data on body mass collected during roost inspections in winter (January-February) and tested for a temporal change in mass. If there has not been a decline in body mass during winter, this may indicate that the birds strategically alter their body weight during chick feeding in response to their work rate, which depends on their timing of reproduction relative to the food peak (Thomas et al. 2001). In contrast, a temporal decline in body mass during the winter months suggests more long-term effects of the mistiming the birds face during the breeding season. We had sufficient data only from the HV and VL populations to test for decline in winter body mass, and the GLMMs showed no decline in the HV population $(b=0.002$, $\left.\mathrm{SE}=0.002, \chi_{1}^{2}=0.91, P=.34\right)$ but a significant decline in the VL population $\left(b=-0.01, \mathrm{SE}=0.002, \chi_{1}^{2}=\right.$ 29.08, $P<.001)$. Thus, in the OH and VL populations, the observed decline in body mass is to some degree also caused by factors outside the breeding season, whereas in the HV population, the decline seems to be taking place during the breeding season only, perhaps as a result of an increase in how hard the birds need to work to find food. These results imply that factors other than temperature are the main agents responsible for the observed decline in body mass in our great tit populations and, more generally, are in agreement with those of other studies that have found temperature to be of minor importance in determining body size clines (e.g., Stillwell et al. 2007).

We conclude that the observed decline in body size in our study populations is not in direct response to an increase in temperature but is due to phenotypic plastic responses to changes in prey dynamics that have led to a decrease in fledgling weight as well as in adult weight. Our results highlight the importance of considering alternative environmental factors covarying with temperature when exploring size clines in the context of Bergmann's rule, and they provide an important example of how a detailed examination of phenotypic, genetic, and environmental patterns can disentangle the causes behind Bergmann's temperature-size clines.

\section{Acknowledgments}

We are grateful to the many people who have collected data during the long-term tit studies; in particular, we would like to thank H. Bouwmeester, L. Holleman, H. van Eck, and J. Visser. The population studies have been conducted under the directorships of H. N. Kluyver (1955-
1968), J. H. van Balen (1968-1991), and A. J. van Noordwijk (1991-2002). The database has been managed by $\mathrm{L}$. Vernooij and J. Visser. Comments from three anonymous reviewers greatly improved the manuscript. We are also grateful to A. Charmantier, E. Immonen, L. Kruuk, D. Nussey, and B. Rogell for comments and discussion that improved the manuscript. J. Hadfield provided helpful assistance with the MCMCglmm package. A.H. was financially supported by a GENACT Project studentship funded by the Marie Curie Host Fellowships for Early Stage training at the University of Edinburgh; a stipend from the Wolfgang Pauli Institute, University of Vienna; and a reintegration grant from the European Commission. S.M.H. is supported by the Wolfgang Pauli Institute and the University of Natural Resources and Applied Sciences in Vienna, and M.E.V. is supported by an NWO (Netherlands Organization for Scientific Research) Vici grant.

\section{Literature Cited}

Adams, D. C., and J. O. Church. 2008. Amphibians do not follow Bergmann's rule. Evolution 62:413-420.

Bergmann, C. 1847. Über die Verhältnisse der wärmeökonomie der Thiere zu ihrer Grösse. Göttinger Studien 3:595-708.

Charmantier, A., and D. Reale. 2005. How do misassigned paternities affect the estimation of heritability in the wild? Molecular Ecology 14:2839-2850.

Cresswell, W., J. A. Clark, and R. Macleod. 2009. How climate change might influence the starvation-predation risk trade-off response. Proceedings of the Royal Society B: Biological Sciences 276:35533560.

Damuth, J. 1981. Population density and body size in mammals. Nature 290:699-700.

Falconer, D. S., and T. F. C. Mackay. 1996. Introduction to quantitative genetics. Longman, Essex.

Garant, D., L. E. B. Kruuk, R. H. McCleery, and B. C. Sheldon. 2004. Evolution in a changing environment: a case study with great tit fledging mass. American Naturalist 164:E115-E129.

Gienapp, P., C. Teplitsky, J. S. Alho, J. A. Mills, and J. Merilä. 2008. Climate change and evolution: disentangling environmental and genetic responses. Molecular Ecology 17:167-178.

Gilmour, A. R., B. J. Gogel, B. R. Cullis, and R. Thompson. 2006. ASReml user guide, release 2.0. VSN International, Hemel Hempstead.

Gosler, A. G. 1993. The great tit. Hamlyn, London.

Hadfield, J. D. 2010. MCMC methods for multi-response generalized linear mixed models: the MCMCglmm R package. Journal of Statistical Software 33:1-22.

Hadfield, J. D., A. J. Wilson, D. Garant, B. C. Sheldon, and L. E. B. Kruuk. 2010. The misuse of BLUP in ecology and evolution. American Naturalist 175:116-125.

Henderson, C. R. 1950. Estimation of genetic parameters. Annals of Mathematical Statistics 21:309-310.

Houle, D. 1992. Comparing evolvability and variability of quantitative traits. Genetics 130:195-204.

Husby, A., L. E. B. Kruuk, and M. E. Visser. 2009. Decline in the frequency and benefits of multiple brooding in great tits as a 
consequence of a changing environment. Proceedings of the Royal Society B: Biological Sciences 276:1845-1854.

Husby, A., D. H. Nussey, M. E. Visser, A. J. Wilson, B. C. Sheldon, and L. E. B. Kruuk. 2010. Contrasting patterns of phenotypic plasticity in reproductive traits in two great tit (Parus major) populations. Evolution 64:2221-2237.

Jensen, H., B.-E. Sæther, T. H. Ringsby, J. Tufto, S. C. Griffith, and H. Ellegren. 2004. Lifetime reproductive success in relation to morphology in the house sparrow Passer domesticus. Journal of Animal Ecology 73:599-611.

Johnston, R. F., and R. K. Selander. 1964. House sparrows: rapid evolution of races in North America. Science 144:548-550.

Kingsolver, J. G., H. E. Hoekstra, J. M. Hoekstra, D. Berrigan, S. N. Vignieri, C. E. Hill, A. Hoang, P. Gibert, and P. Beerli. 2001. The strength of phenotypic selection in natural populations. American Naturalist 157:245-261.

Kruuk, L. E. B. 2004. Estimating genetic parameters in natural populations using the "animal model." Philosophical Transactions of the Royal Society B: Biological Sciences 359:873-890.

Kruuk, L. E. B., J. Merilä, and B. C. Sheldon. 2001. Phenotypic selection on a heritable size trait revisited. American Naturalist 158:557-571.

Lande, R., and S. J. Arnold. 1983. The measurement of selection on correlated characters. Evolution 37:1210-1226.

Laugen, A. T., A. Laurila, K. I. Jonsson, F. Söderman, and J. Merilä. 2005. Do common frogs (Rana temporaria) follow Bergmann's rule? Evolutionary Ecology Research 7:717-731.

Lubjuhn, T., S. Strohbach, J. Brun, T. Gerken, and J. T. Epplen. 1999. Extra-pair paternity in great tits (Parus major): a long term study. Behaviour 136:1157-1172.

Lynch, M., and B. Walsh. 1998. Genetics and analysis of quantitative traits. Sinauer, Sunderland, MA.

Mayr, E. 1956. Geographical character gradients and climatic adaptation. Evolution 10:105-108.

McAdam, A. G., and S. Boutin. 2003. Effects of food abundance on genetic and maternal variation in the growth rate of juvenile red squirrels. Journal of Evolutionary Biology 16:1249-1256.

Merilä, J., and B. C. Sheldon. 2001. Avian quantitative genetics. Pages 179-255 in J. Van Nolan and E. D. Ketterson, eds. Current ornithology. Vol. 16. Kluwer Academic, New York.

Merilä, J., L. E. B. Kruuk, and B. C. Sheldon. 2001. Cryptic evolution in a wild bird population. Nature 412:76-79.

Millien, V. 2004. Relative effects of climate change, isolation and competition on body-size evolution in the Japanese field mouse, Apodemus argenteus. Journal of Biogeography 31:1267-1276.

Millien, V., and J. Damuth. 2004. Climate change and size evolution in an island rodent species: new perspectives on the island rule. Evolution 58:1353-1360.

Milner, J. M., S. D. Albon, A. W. Illius, J. M. Pemberton, and T. H. Clutton-Brock. 1999. Repeated selection of morphometric traits in the Soay sheep on St. Kilda. Journal of Animal Ecology 68:472-488.

Morrissey, M. B., and A. J. Wilson. 2010. pedantics: an R package for pedigree-based genetic simulation and pedigree manipulation, characterization and viewing. Molecular Ecology Resources 10: 711-719.

Parmesan, C. 2006. Ecological and evolutionary responses to recent climate change. Annual Review of Ecology, Evolution, and Systematics 37:637-669.

Partridge, L., and J. A. Coyne. 1997. Bergmann's rule in ectotherms: is it adaptive? Evolution 51:632-635.
Perdeck, A. C., M. E. Visser, and J. H. van Balen. 2000. Great tit Parus major survival, and the beech-crop cycle. Ardea 88:99-108.

Postma, E. 2006. Implications of the difference between true and predicted breeding values for the study of natural selection and micro-evolution. Journal of Evolutionary Biology 19:309-320.

R Development Core Team. 2007. R: a language and environment for statistical computing. R Foundation for Statistical Computing, Vienna. http://www.R-project.org.

Salewski, V., W. M. Hochachka, and W. Fiedler. 2010. Global warming and Bergmann's rule: do central European passerines adjust their body size to rising temperatures? Oecologia (Berlin) 162:247-260.

Schluter, D., and J. N. M. Smith. 1986. Natural selection on beak and body size in the song sparrow. Evolution 40:221-231.

Scholander, P. F. 1955. Evolution of climatic adaptation in homeotherms. Evolution 9:15-26.

Smith, F. A., J. L. Betancourt, and J. H. Brown. 1995. Evolution of body size in the woodrat over the past 25,000 years of climate change. Science 270:2012-2014.

Stillwell, R. C., G. E. Morse, and C. W. Fox. 2007. Geographic variation in body size and sexual size dimorphism of a seed-feeding beetle. American Naturalist 170:358-369.

Teplitsky, C., J. A. Mills, J. S. Alho, J. W. Yarrall, and J. Merilä. 2008. Bergmann's rule and climate change revisited: disentangling environmental and genetic responses in a wild bird population. Proceedings of the National Academy of Sciences of the USA 105: 13492-13496.

Thomas, D. W., J. Blondel, P. Perret, M. M. Lambrechts, and J. R. Speakman. 2001. Energetic and fitness costs of mismatching resource supply and demand in seasonally breeding birds. Science 291:2598-2600.

van Balen, J. H. 1973. Comparative study of breeding ecology of great tit (Parus major) in different habitats. Ardea 61:1-93.

Verboven, N., and A. C. Mateman. 1997. Low frequency of extrapair fertilizations in the great tit Parus major revealed by DNA fingerprinting. Journal of Avian Biology 28:231-239.

Visser, M. E., A. J. van Noordwijk, J. M. Tinbergen, and C. M. Lessells. 1998. Warmer springs lead to mistimed reproduction in great tits (Parus major). Proceedings of the Royal Society B: Biological Sciences 265:1867-1870.

Visser, M. E., L. J. M. Holleman, and P. Gienapp. 2006. Shifts in caterpillar biomass phenology due to climate change and its impact on the breeding biology of an insectivorous bird. Oecologia (Berlin) 147:164-172.

Watt, C., S. Mitchell, and V. Salewski. 2010. Bergmann's rule; a concept cluster? Oikos 119:89-100.

Wood, S. 2006. Generalized additive models: an introduction with R. Chapman \& Hall/CRC, Boca Raton, FL.

Yom-Tov, Y. 2001. Global warming and body mass decline in Israeli passerine birds. Proceedings of the Royal Society B: Biological Sciences 268:947-952.

Yom-Tov, Y., and S. Yom-Tov. 2006. Decrease in body size of Danish goshawks during the twentieth century. Journal of Ornithology 147:644-647.

Yom-Tov, Y., S. Yom-Tov, J. Wright, C. J. R. Thorne, and R. Du Feu. 2006. Recent changes in body weight and wing length among some British passerine birds. Oikos 112:91-101.

Associate Editor: Dean C. Adams Editor: Mark A. McPeek 